\title{
Resistência de união à dentina de quatro sistemas adesivos
}

\section{Bond strength of four adhesive systems to dentin}

\author{
Marcela Rocha de Oliveira Carrilho* \\ Alessandra Reis** \\ Alessandro Dourado Loguercio** \\ Leonardo Eloy Rodrigues Filho***
}

\begin{abstract}
RESUMO: O objetivo do presente estudo foi avaliar a resistência adesiva de quatro sistemas adesivos, composicionalmente diferentes, aplicados à dentina humana. Doze dentes terceiros molares humanos tiveram o esmalte oclusal removido para exposição de uma superficie plana de dentina, na qual foram realizados os procedimentos de adesão. Os dentes foram aleatoriamente divididos em quatro grupos, considerando-se o sistema adesivo e a resina composta a serem empregados: Grupo 1 - Single Bond + P60 (SB); Grupo 2 - Bond 1 + Surefil (B1); Grupo 3 - Prime \& Bond NT + Alert (NT) e Grupo 4 - Prime \& Bond 2.1 + TPH (2.1). Após 24 h de armazenagem em água destilada a $37^{\circ} \mathrm{C}$, os dentes foram seccionados, longitudinalmente, em cortes perpendiculares entre si, para que fossem obtidos espécimes em formato de um paralelogramo com secção transversal retangular de $0,8 \mathrm{~mm}^{2}$ de área e $10 \mathrm{~mm}$ de comprimento, em média. Os espécimes foram submetidos ao teste de microtração. A análise de variância $(\alpha=0,05)$ demonstrou não haver diferença significante entre os valores médios de resistência obtidos pelos quatro adesivos, embora a análise dos espécimes que sofreram fratura precoce tenha evidenciado menor sensibilidade para o sistema SB.
\end{abstract}

UNITERMOS: Dentina; Adesivos dentinários; Resistência à tração.

\begin{abstract}
The purpose of the present study was to evaluate the bond strength of four adhesive systems to dentin. Twelve human third molars had their occlusal enamel removed in order to expose a flat dentinal surface, on which the adhesive procedures were carried out. The teeth were divided into four groups, according to the employed adhesive system and composite resin: Group 1 - Single Bond + P60 (SB); Group 2 - Bond 1 + Surefil (B1); Group 3 - Prime \& Bond NT + Alert (NT); and Group $4-$ Prime $\&$ Bond $2.1+$ TPH (2.1). After $24 \mathrm{~h}$ in distilled water at $37^{\circ} \mathrm{C}$, the teeth were longitudinally sectioned in two perpendicular directions in order to obtain parallelogram-shaped specimens with a cross-sectional area of $0.8 \mathrm{~mm}^{2}$ and $10 \mathrm{~mm}$ of length, on the average. The test specimens were submitted to microtensile test. The data were submitted to ANOVA $(\alpha=0.05)$, which revealed no differences between the groups, although the analysis of the specimens that presented early fracture evidenced the lower sensitivity of the SB system. UNITERMS: Dentin; Dentin-bonding agents; Tensile strength.
\end{abstract}

\section{INTRODUÇÃO}

O sucesso clínico de uma restauração, de qualquer natureza, baseia-se sobretudo no selamento que o material restaurador proporciona às margens do preparo cavitário. No caso das restaurações em que se utiliza associação de resinas compostas e sistemas adesivos, o bom selamento estará muitas vezes restrito à capacidade que o material apresenta em resistir aos esforços mecânicos imediatos, decorrentes de seu próprio mecanismo de cura, ou tardios devido às ações fisiopatológicas do aparelho estomatognático.

Dessa forma, pesquisar o comportamento fisico-mecânico das interfaces estabelecidas pelos sistemas adesivos e o substrato dentário consti- tui-se recurso importante que, somado a outros experimentos in vitro e in vivo, contribui com a elaboração de um prognóstico restaurador, aceitável ou não, sobretudo, quando se considera o número extenso de materiais disponíveis, bem como a velocidade com que são lançados e retirados do mercado, não havendo, às vezes, tempo para que sua performance seja criteriosamente avaliada.

Quando os ensaios mecânicos laboratoriais são empregados com esta finalidade, nota-se que, na maioria dos estudos, os testes de cisalhamento e tração têm sido os instrumentos metodológicos mais utilizados para aferição da resistência adesiva, tanto à dentina quanto ao esmalte ${ }^{1}$. Com o uso dos sistemas adesivo atuais, associado às técnicas

\footnotetext{
*Doutoranda em Materiais Dentários; ***Professor Doutor do Departamento de Materiais Dentários - Faculdade de Odontologia da Universidade de São Paulo.

** Professores Doutores do Departamento de Materiais Dentários e Dentística Operatória da Universidade do Oeste de Santa Catarina - Joaçaba.
} 
Carrilho MR de O, Reis A, Loguercio AD, Rodrigues Filho LE. Resistência de união à dentina de quatro sistemas adesivos. Pesqui Odontol Bras 2002;16(3):251-256.

de condicionamento total e adesão à dentina úmida, duas evidências passaram a ser registradas: aumento tanto dos valores de resistência adesiva ${ }^{14}$ quanto do número das fraturas coesivas em dentina, permanecendo a interface intacta ${ }^{15,18}$.

A ocorrência destas fraturas coesivas poderia induzir o raciocínio de que, enfim, havia se desenvolvido materiais que estariam promovendo uma adesão tão ou mais resistente do que a própria resistência coesiva da dentina. Entretanto, alguns centros de pesquisa advogam que, embora se tenha aumentado o valor nominal da resistência adesiva promovida pelo uso dos novos sistemas adesivos, o aumento do número de fraturas coesivas seria devido à distribuição não homogênea das tensões, durante a realização dos testes mecânicos ${ }^{14,18,20,21}$.

A redução da área adesiva, para valores menores do que aqueles utilizados nos testes mecânicos usuais, é fator que permite, por exemplo, melhorar a distribuição das tensões na interface ${ }^{3,7,18}$. Com base neste princípio, o teste de microtração tem proposto a utilização de áreas que giram em torno de 0,8-1 $\mathrm{mm}^{2} \mathrm{e}$, assim, possibilitam a obtenção de falhas quase que exclusivamente adesivas, permitindo uma análise da real resistência de união entre o material e a estrutura dentária ${ }^{6,15,16,17}$.

Dessa forma, foi proposta da presente pesquisa avaliar a resistência de união formada pela dentina e por 4 sistemas adesivos de uso corrente, por meio do ensaio de microtração.

\section{MATERIAL E MÉTODO Preparo dos dentes}

Este estudo foi aprovado pelo Comitê de Ética em Pesquisa da FOUSP $\left(n^{\circ} 34\right)$. Foram utilizados 12 terceiros molares humanos integros, extraídos por razões ortodônticas, armazenados em solução de cloramina a $0,5 \%$. O esmalte oclusal foi removido, por meio de um disco de diamante montado à máquina de corte Labcut 1010 (Extec, EUA). As superficies de dentina expostas foram desgastadas e planificadas em lixa de $\mathrm{SiC} \mathrm{n}^{\circ} 600$ (1 minuto), para gerar um padrão de camada de "smear" semelhante em todos os dentes.

\section{Procedimentos de adesão}

Os dentes foram aleatoriamente divididos em quatro grupos, sumarizados no Quadro 1, no qual se encontram listados os sistemas adesivos e as resinas compostas utilizadas.

Após o condicionamento, com ácido fosfórico $37 \%(15 \mathrm{~s})$, as superficies foram lavadas (15 s), secas com jato de ar e re-umedecidas com $4 \mu \mathrm{lde}$ água. Dada a variação das áreas destinadas à adesão, e considerando-se o tipo de solvente presente no adesivo a ser utilizado, em certos casos houve necessidade de remoção do excesso de água, que foi feito com papel absorvente (filtro de papel para café, Melita ${ }^{\circledast}$, Brasil), recortado em perfurador de papel com $13 \mathrm{~mm}^{2}$ de área.

Para superficies de dentina com cerca de $50 \mathrm{~mm}^{2}$ de área, por exemplo, foram utilizados, em média, de 1 a 2 e de 3 a 4 papéis absorventes quan-

QUADRO 1 - Relação dos grupos de estudo.

\begin{tabular}{||c|l|l||}
\hline \hline Grupo & \multicolumn{1}{|c|}{ Sistema adesivo } & \multicolumn{1}{|c||}{ Resina composta } \\
\hline 1 & $\begin{array}{l}\text { Single Bond (SB)* - Composição: Bis-GMA, HEMA, re- } \\
\text { sinas dimetacrilatos, copolímeros do ácido polialquenói- } \\
\text { co, água e etanol. }\end{array}$ & $\begin{array}{l}\text { P60 (P60)* - Composição: Bis-GMA, UDMA, Bis-EMA, } \\
\text { silicato de zircônia. }\end{array}$ \\
\hline 2 & $\begin{array}{l}\text { Bond 1 (B1)** - Composição: HEMA, Bis-GMA, água, } \\
\text { acetona, etil 4-dimetil amino benzoato, hidroxibutil tolue- } \\
\text { no, ácido pirometil/glicoldimetacrilato e iniciadores. }\end{array}$ & $\begin{array}{l}\text { Surefil (SUR)** - Composição: UDMA, vidro de } \\
\text { Si-Bo-Al-F Ba, SiO, e nanopartículas. }\end{array}$ \\
\hline 3 & $\begin{array}{l}\text { Prime \& Bond NT (NT)*** - Composição: resinas di- e } \\
\text { trimetacrilato, sílica amorfa, PENTA, fotoiniciadores, es- } \\
\text { tabilizadores, cetilamina hidrofluorada e acetona. }\end{array}$ & $\begin{array}{l}\text { Alert (ALE)*** - Composição: Bis-GMA etoxilatado, vi- } \\
\text { dro de Ba-Al-Si, SiO, e carga vítrea filamentar. }\end{array}$ \\
\hline 4 & $\begin{array}{l}\text { Prime \& Bond 2.1 (PB)**** - Composição: resinas di- e } \\
\text { trimetacrilato, PENTA, hidroxitolueno butilato, 4 etildi- } \\
\text { metil amino benzoato, fotoiniciadores, cetilamina hidro- } \\
\text { fluorada e acetona. }\end{array}$ & $\begin{array}{l}\text { TPH (Spectrum) (TPH)**** Composição: Bis-GMA e } \\
\text { UDA, silicato de bário. }\end{array}$ \\
\hline
\end{tabular}

*3M Dental, St. Paul, EUA; **Jeneric/Pentron, Wallingford, EUA; ***Dentsply De Trey, Konstanz, Alemanha; $* * * *$ Dentsply Caulk, Milford, EUA. 
Carrilho MR de O, Reis A, Loguercio AD, Rodrigues Filho LE. Resistência de união à dentina de quatro sistemas adesivos. Pesqui Odontol Bras 2002;16(3):251-256.

do da aplicação, respectivamente, dos adesivos a base de acetona e a base de água/etanol. As superficies que receberam os adesivos a base de acetona (grupos 2, 3 e 4) foram mantidas, portanto, mais úmidas do que aquelas em que o adesivo empregado continha, como solvente, uma mistura de água e etanol (grupo 1).

Os sistemas a base de acetona foram aplicados na superficie de dentina sem agitação, ao contrário do sistema a base de água/etanol que foi aplicado ativamente, esfregando-se o pincel por toda superficie. O excesso de solvente foi removido com jato de ar e após a fotoativação do adesivo, a resina composta correspondente foi adicionada em 2 camadas $( \pm 2 \mathrm{~mm})$, fotoativada, individualmente, por 40 segundos numa intensidade de $600 \mathrm{~mW} / \mathrm{cm}^{2}$ (Optilux 400, Demetron, EUA).

Todos os dentes foram armazenados por 24 horas em água destilada à temperatura de $37^{\circ} \mathrm{C}$.

\section{Obtenção dos espécimes}

De cada dente foram obtidos, por cortes seriados e perpendiculares entre si, espécimes com
$10 \mathrm{~mm}$ de comprimento e área de secção transversal retangular de, aproximadamente, $0,8 \mathrm{~mm}^{2}$ (Figura 1).

\section{Ensaio de microtração}

Cada espécime foi fixado, com cola a base de cianoacrilato (Zapit, DVA, EUA), à garra desenvolvida por Bianchi ${ }^{3}$ (1999), e testado na máquina universal de ensaios Kratos (Kratos Dinamômetros, Brasil) à velocidade de $0,5 \mathrm{~mm} / \mathrm{min}$ (Figura 2). O modo de fratura de cada espécime foi observado em lupa estereoscópica (Olympus SZ40, Japão) e a área da secção transversal foi mensurada por paquímetro digital (Absolute Digimatic, Mitutoyo, Japão) na região próxima à fratura, para que fosse feito o cálculo da resistência em MPa.

\section{Análise estatística}

Os dados de resistência adesiva foram submetidos à análise de variância de um critério, cuja fonte de variação foi o fator sistema adesivo, em quatro niveis $(\alpha=0,05)$. Foi também aplicado o teste qui-quadrado $(\alpha=0,05)$ para comparação do per-
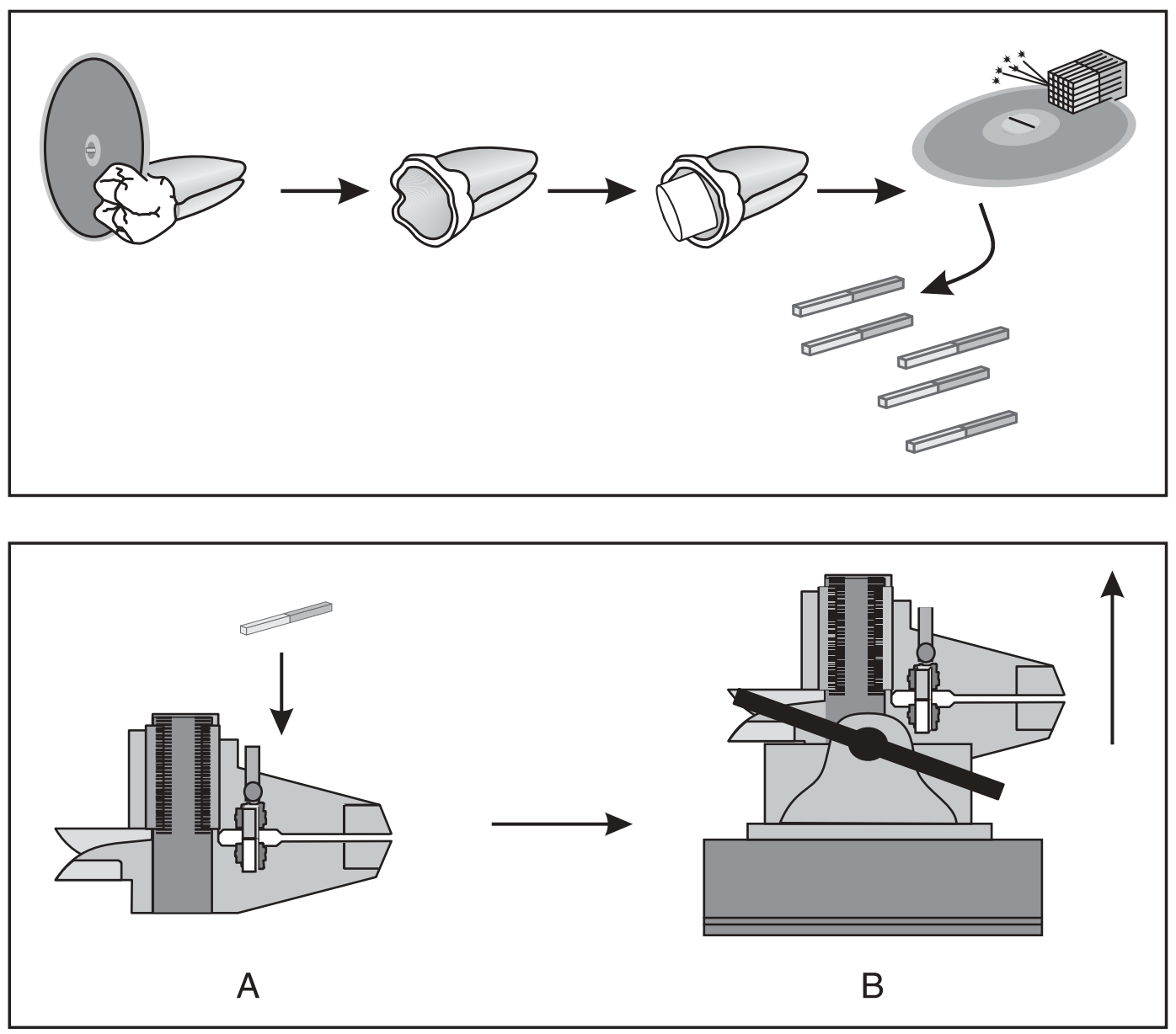

FIGURA 1 - Esquema da seqüência de preparo e obtenção dos corpos-de-prova. Da esquerda para direita: remoção do esmalte oclusal; exposição da superficie plana de dentina; realização dos procedimentos de adesão; realização dos cortes perpendiculares entre si para obtenção dos corpos-de-prova em forma de paralelogramo.

FIGURA 2 - A: união do espécime à garra com cola; B: fixação da garra à maquina de ensaio e realização do ensaio de tração na direção axial à interface de união. 
Carrilho MR de O, Reis A, Loguercio AD, Rodrigues Filho LE. Resistência de união à dentina de quatro sistemas adesivos. Pesqui Odontol Bras 2002;16(3):251-256.

TABELA 1 - Média de resistência em MPa e desvio padrão (M $\pm \mathrm{DP})$, e percentual de espécimes fraturados antes que o teste tivesse sido realizado (adesão zero).

\begin{tabular}{c|c|c}
\hline \hline Grupo & $\mathrm{M} \pm \mathrm{DP}$ & Adesão zero $(\%$ do total $)$ \\
\hline 1 (SB + P60) & $35,6 \pm 15,0 \mathrm{a}$ & $6(8,3) \mathrm{b}$ \\
\hline $2(\mathrm{~B} 1+\mathrm{SUR})$ & $33,3 \pm 11,6 \mathrm{a}$ & $25(64,1) \mathrm{c}$ \\
\hline $3(\mathrm{NT}+\mathrm{ALE})$ & $28,0 \pm 8,50 \mathrm{a}$ & $17(52,1) \mathrm{c}$ \\
\hline $4(\mathrm{~PB}+\mathrm{TPH})$ & $34,8 \pm 12,4 \mathrm{a}$ & $23(44,23) \mathrm{c}$ \\
\hline \hline
\end{tabular}

Os dados acompanhados por letras iguais foram estatisticamente semelhantes.

centual de espécimes fraturados antes que o ensaio mecânico tivesse sido realizado.

\section{RESULTADOS}

A análise descritiva dos dados, expressos em valores de média e desvio padrão, está representada na Tabela 1. A análise de variância de um critério revelou não haver diferença significante entre os valores de resistência apresentados nos quatro niveis do fator material $(\mathrm{p}>0,05)$.

O teste qui-quadrado, entretanto, detectou que o percentual de espécimes que sofreu fratura precoce, ou seja, antes que o ensaio mecânico tivesse sido realizado, foi significantemente menor para o grupo $1(\mathrm{p}<0,05)$ e similar para os grupos 2,3 e 4 $(p>0,05)$ (Tabela 1).

O modo de fratura predominante, considerando-se todos os espécimes, foi adesivo, entre o sistema adesivo e a dentina (63\%); seguido do modo misto em que parte da fratura foi adesiva, entre o sistema adesivo e a dentina, e parte coesiva no adesivo (34\%). Os demais modos de fratura foram coesivo em resina $(2 \%)$ e coesivo em dentina $(1 \%)$.

\section{DISCUSSÃO}

Considerando-se os valores de resistência alcançados (Tabela 1) o que se observa é a tendência de classificar semelhantemente o desempenho dos diferentes sistemas adesivos. De certo modo, esta ocorrência pode parecer surpreendente, já que os materiais empregados têm composição bastante diferente (Quadro 1), fato considerado relevante na magnitude da resistência adesiva à dentina ${ }^{8}$. Entretanto, parece oportuno avaliar os resultados não apenas sob o aspecto nominal dos valores de resistência, mas também sob aspectos que podem revelar a diferenciação de um sistema em relação a outro, como por exemplo à análise dos espécimes que sofreram fratura sem que tivessem sido submetidos ao teste de microtração.

Um fator de importância fundamental à hibridização da dentina condicionada, e conseqüentemente à qualidade da adesão, é a umidade do substrato aderente, que deve ser específica para o tipo de adesivo empregado ${ }^{9,11,19}$. Quando a acetona, utilizada como veículo de sistemas adesivos, entra em contato com a dentina úmida há um aumento intenso da pressão de vapor da água remanescente ${ }^{9}$ e, se esta não estiver em quantidade suficientemente elevada, rapidamente a dentina terá sua permeabilidade diminuída, dificultando assim a penetração dos monômeros resinosos ${ }^{13}$. Outro aspecto que colabora com o aumento da velocidade de evaporação da água é a agitação do adesivo sobre a superfície dentinária .

Assim, de outro modo, adesivos que têm como solvente uma mistura de água e etanol, que não alteram tão abruptamente a pressão de vapor da água, podem ter seu desempenho prejudicado quando aplicados em substratos mais úmidos e sem agitação constante ${ }^{10}$. Neste estudo, a obtenção da umidade dentinária compativel com o solvente presente em cada sistema adesivo utilizado foi favorecida pelas condições do substrato (dentina planificada), e do protocolo seguido, que permitiu trabalhar com uma quantidade de água predeterminada, que de certo modo pode ter conduzido à semelhança estatística dos valores de resistência atingidos pelos materiais.

Um outro fator que parece contribuir com a similaridade estatística dos valores encontrados reside no fato de os procedimentos de preparo superficial e adesão terem sido conduzidos por um único operador, previamente adestrado para uso de todos os materiais empregados. Embora a intenção do estudo não ter sido a calibração do operador, a realização de trabalhos prévios parece ter capacitado o mesmo à utilização destes materiais. Conforme atestam Miyasaki et al. ${ }^{12}$ (2000), uma grande variabilidade nos valores de resistência adesiva pode ser atribuída ao grau de conhecimento e familiarização dos diferentes operadores com os procedimentos e materiais empregados.

Os valores de resistência adesiva registrados com qualquer um dos sistemas adesivos testados foram sempre altos, assemelhando-se àqueles relatados por outros autores, que utilizaram o teste de microtração para testar os mesmos materiais $^{5,6,17}$, mas diferem radicalmente dos valores obtidos quando empregados os testes de cisalhamento ou tração convencional ${ }^{2,5,17,22}$. 
Carrilho MR de O, Reis A, Loguercio AD, Rodrigues Filho LE. Resistência de união à dentina de quatro sistemas adesivos. Pesqui Odontol Bras 2002;16(3):251-256.

É preciso ressaltar que os espécimes preparados para os testes de cisalhamento e tração convencional apresentam uma área de adesão com secção transversal, em média, dez vezes maior do que a área de um espécime preparado para o teste de microtração. Em áreas maiores aumentam-se as chances de que o procedimento adesivo seja feito em substrato mais heterogêneo e conseqüentemente, que este contenha maior número de defeitos, intrinsecamente relacionados ao processo de $\operatorname{adesão}^{14,18}$. A concentração de tensões, ao redor destes defeitos, poderá ocasionar fraturas com valores de resistência mais baixos ${ }^{4}$. Com o teste de microtração, a probabilidade de que a interface de união contenha defeitos é menor, aumentando-se, portanto, os valores nominais de resistência ${ }^{16}$.

Entretanto, antes que os espécimes de microtração sejam obtidos pelos cortes seriados, o procedimento adesivo é realizado numa extensa área de dentina planificada. Pelo mesmo raciocínio que se desenvolveu para justificar os menores valores de resistência dos espécimes de cisalhamento e tração convencional, supõe-se que nesta extensa área de dentina haverá presença de defeitos também nos espécimes de microtração. Ao cortar o dente para que vários espécimes sejam originados, existirá desde aqueles que não conterão defeito algum, até aqueles cujo defeito poderá representar quase que a totalidade da interface, e estes poderão se fraturar antes que o ensaio mecânico seja realizado.

Cabe destacar, portanto, que se por um lado os valores de resistência obtidos foram semelhantes, classificando os materiais num mesmo patamar de efetividade, por outro lado, o percentual de espécimes fraturados, sem que tivesse sido realizado o teste de microtração, não posiciona estes materiais similarmente. Neste quesito, o adesivo SB foi significantemente melhor, com apenas $8 \%$ de falhas precoces, enquanto os demais apresentaram percentuais altos de falha, respectivamente $64 \%$ para B1, 52\% para NT e $44 \%$ para PB, o que de certa forma sinaliza maior sensibilidade destes materiais. É possivel, ainda, admitir que tal distinção só tenha sido verificada pelo emprego do teste de microtração, que permite testar vários espécimes provenientes de único dente, diferentemente dos testes de cisalhamento e tração convencional que proporcionam uma única área de adesão.

Parece fundamental, portanto, que os trabalhos, que utilizam testes mecânicos para verificação da resistência adesiva de diferentes materiais, abordem não só a comparação de valores obtidos, como também a avaliação de outros parâmetros como modo de fratura preponderante e o percentual de fraturas precoces, de forma a complementar a análise do estudo.

\section{CONCLUSÃO}

É lícito concluir que o sistema SB tenha, nas condições deste estudo, demonstrado menor sensibilidade garantida pelo baixo percentual de espécimes fraturados precocemente, embora a resistência observada tenha sido semelhante para todos os adesivos.

\section{AGRADECIMENTOS}

Os autores agradecem à CAPES e à FAPESP (99/10043-0 e 99/05124-0) pelo suporte financeiro; aos Srs. Paulo Eduardo dos Santos e Antonio Carlos Lascala pelas ilustrações.

\section{REFERÊNCIAS}

1. Al-Salehi SK, Burke FJT. Methods used in dentin bonding tests: an analysis of 50 investigations on bond strength. Quintessence Int 1997;28(11):717-23.

2. Al-Ehaideb A, Mohammed H. Shear bond strength of "one-bottle" dentin adhesives. J Prosthet Dent 2000;84(4):408-12.

3. Bianchi J. Estudo sobre a resistência à microtração em função das dimensões, modo de preensão e formato do corpo-de-prova. (Tese de Doutorado). São Paulo: Universidade de São Paulo, Faculdade de Odontologia; 1999.

4. Bianchi J, Rodrigues Filho LE, Santos JFF. Resistência adesiva de resinas compostas à dentina. Rev Odontol Univ São Paulo 1999;13(1):51-5.

5. Cardoso PEC, Braga RR, Carrilho MRO. Evaluation of micro-tensile, shear and tensile tests determining the

bond strength of three adhesive systems. Dent Mater 1998;14(6):394-8.

6. Cardoso PEC, Carrilho MRO, Francci CE, Perdigão J. Microtensile bond strength of one-bottle dentin adhesives. Am J Dent 2001;14(1):22-4.

7. DeHoff PH, Anusavice KJ, Wang Z. Three-dimensional finite element analysis of shear bond test. Dent Mater 1995; 11(2):126-31

8. Eick JD, Gwinnett AJ, Pashley DH, Robinson SJ. Current concepts on adhesion to dentin. Crit Rev Oral Biol Med 1997;8(3):306-35.

9. Jacobsen T, Söderholm KJ. Effect of primer solvent, primer agitation, and dentin dryness on shear bond strength to dentin. Am J Dent 1998;11(5):225-8.

10. Jacobsen T, Söderholm KJ. Some effects of water on dentin bonding. Dent Mater 1995;11(2):132-6. 
Carrilho MR de O, Reis A, Loguercio AD, Rodrigues Filho LE. Resistência de união à dentina de quatro sistemas adesivos. Pesqui Odontol Bras 2002;16(3):251-256.

11. Kanca J. Effect of resin primer solvents and surface wetness on resin composite bond to strength. Am J Dent 1992;5(4):213-5.

12. Miyasaki M, Onose H, Moore KB. Effect of operator variability on dentin bond strength of two-step bonding systems. Am J Dent 2000;13(2):101-4.

13. Pashley DH, Agee KA, Nakajima M, Tay FR, Carvalho RM, Terada RS, et al. Solvent-induced dimensional changes in EDTA-demineralized dentin matrix. J Biomed Mater Res 2001;56(2): 273-81.

14. Pashley DH, Carvalho RM, Sano H, Nakajima M, Yoshiyama M, Shono Y, et al. The microtensile bond test: a review. J Adhesive Dent 1999;1(4):299-309.

15. Pashley DH, Sano H, Ciucchi B, Yoshiyama M, Carvalho RM. Adhesion testing of dentin bonding agents: a review. Dent Mater 1995;11(2):117-25.

16. Phrukkanon S, Burrow MF, Tyas MJ. The influence of cross-sectional shape and surface area on the microtensile bond strength. Dent Mater 1998;14(3):212-21.
17. Schreiner R, Chappell RP, Glaros AG, Eick JD. Microtensile test of dentin adhesives. Dent Mater 1998;14(3):194-201.

18. Sundsangiam S, Van Noort R. Do dentin bond strength tests serve a useful purpose? J Adhesive Dent 1999;1(1):57-67.

19. Tay FR, Gwinnett JA, Wei SH. Relation between water content in acetone/alcohol-based primer and interfacial ultrastructure. J Dent 1998; 26(2):147-56.

20. Van Noort R, Norozzi S, Howard IC, Cardew G. A critique of bond strength measurements. J Dent Res 1989;76(2):61-7.

21. Van Noort R, Cardew GH, Howard IC, Norozzi S. The effect of local interfacial geometry on the measurement of tensile bond strength to dentin. J Dent Res 1991;70(5):889-93.

22. Vargas MA, Cobb DS, Denehy GE. Interfacial micromorphology and shear bond strength of single-bottle primer/adhesives. Dent Mater 1997;13(5):316-24.

\section{CD "18 anos de História - Memória Científica da SBPqO"}

\section{Encontra-se à disposição de todos os associados o CD "18 anos de História - Memória Científica da Sociedade Brasileira de Pesquisa Odontológica - 1984/2001".}

Informações: Secretaria da

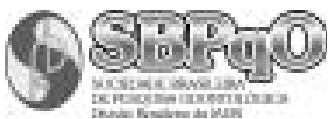

Tel.: (0**11) 3091-7855 e-mail: sbpqo@sbpqo.org.br

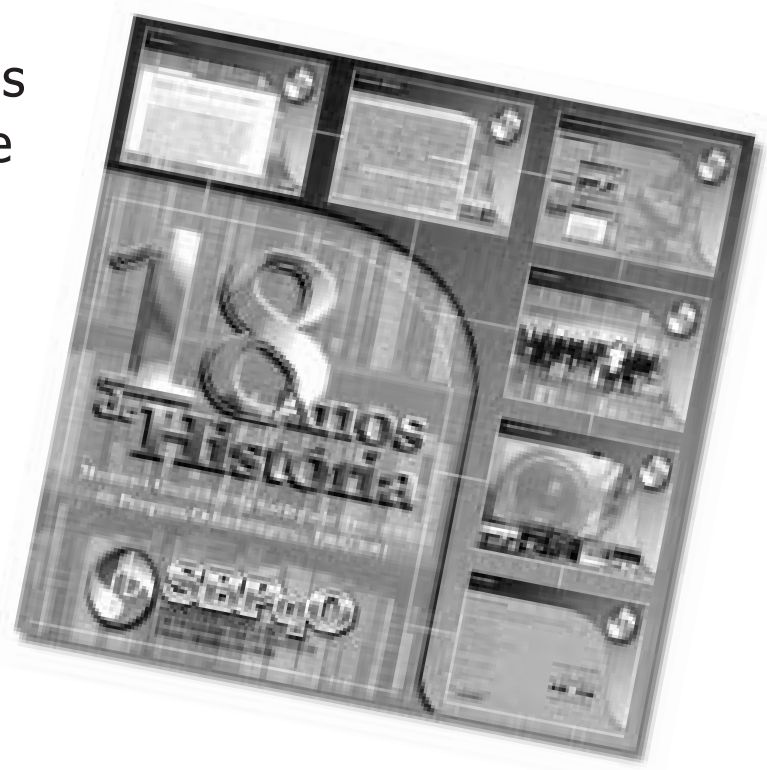

